\title{
Algoritmo Multirecombinativo para la Planificación Dinámica del Mantenimiento de Locaciones Petroleras
}

\author{
Andrea Villagra, Eugenia De San Pedro, Marta Lasso y Daniel Pandolfi \\ Universidad Nacional de la Patagonia Austral, Unidad Académica Caleta Olivia, Laboratorio de \\ Tecnologías Emergentes, Acceso Norte, Ruta No 3 s/n, (9011) Caleta Olivia, Santa Cruz-Argentina \\ e-mail: \{avillagra, edesanpedro, mlasso,dpandolfi\}@uaco.unpa.edu.ar
}

\begin{abstract}
Resumen
Se presenta el desarrollo de una herramienta, denominada PAE (Planificación basada en un Algoritmo Evolutivo) que mejore las planificaciones dinámicas del mantenimiento de locaciones petroleras. Esta herramienta utiliza como motor de planificación un algoritmo evolutivo multirecombinativo que es el generador de múltiples soluciones a este problema. Se compararon los resultados obtenidos con información de planificación histórica facilitada por una empresa. Los resultados han sido satisfactorios y se puede concluir que con el uso de esta herramienta se mejora el tiempo total de la planificación original y se permite la re.planificación dinámica manteniendo el mismo nivel de calidad en los resultados. Finalmente el beneficio puede observarse desde dos puntos de vista: la disminución del costo de mantenimiento y la disminución de la probabilidad de caída o contingencias en la producción.
\end{abstract}

Palabras clave: locaciones petroleras, algoritmo evolutivo, planificación, mantenimiento

\section{Multirecombinative Algorithm for Dynamic Scheduling of Maintenance of Oil Fields}

\begin{abstract}
The development of a computer tool named PAE (Scheduling based on an Evolutionary Algorithm) that improves the dynamic schedules of the maintenance of oil fields, is presented. This tool uses a multirecombinative evolutionary algorithm generator of multiple solutions to this problem. The results were compared with information of historical schedules facilitated by the company. The obtained results have been satisfactory concluding that the use of this tool improves the total timing of the original schedule and dynamic re-scheduling is allowed keeping the same quality level in the results. Finally, the benefit of this should be observed from two points of view: the decrease of the maintenance cost and the reduction of the probability of falling or contingencies in the production.
\end{abstract}

Keywords: oil fields, evolutionary algorithm, scheduling, maintenance 


\section{INTRODUCCIÓN}

Las actividades petroleras de explotación y transporte del petróleo son muy importantes para el desarrollo económico de la sociedad industrial moderna. Sin embargo, estas actividades son generadoras de riesgos que se traducen en contaminaciones accidentales que afectan directamente al ecosistema. El impacto ambiental ocasionado por la industria petrolera comprende los efectos de todas y cada una de las fases involucradas en las etapas tanto de exploración, explotación, transformación, distribución y comercialización. En muchas de las regiones donde se ha explotado el petróleo, se han generado contingencias de diferentes magnitudes como consecuencia de las operaciones desarrolladas en: tanques de almacenamiento, oleoductos, equipos de perforación, buques petroleros, etc. Además de los impactos generados en el medio como consecuencia de la exploración y explotación del petróleo, existen también daños ocasionados a las personas que lo emplean o se encuentran en contacto con él dentro de su ámbito laboral.

En la última década, las Empresas dedicadas a la explotación, producción y transporte del recurso, han visto la necesidad de implementar medidas de prevención a fin de evitar y / o minimizar los daños ocasionados al medio ambiente, personas y bienes materiales.

La mejor manera de atacar el problema de contaminación por petróleo es prevenir el incidente. Normalmente éstos se producen por fallas de equipos o del material y fallas humanas. Los primeros pueden ser subsanados mediante inspecciones periódicas y un mantenimiento adecuado; y los segundos, mediante la instrucción y el entrenamiento del personal en forma permanente. La minimización del tiempo entre cada período de inspección de las locaciones se obtiene con una mejora en la planificación del mantenimiento y esto puede ser abordado como un problema de scheduling. Se ha demostrado, que muchos problemas de scheduling pertenecen a la clase NP-hard (Brucker, 2004) reflejando así la relevancia industrial de este tipo de problemas.

Los Algoritmos Evolutivos (AEs) han sido aplicados exitosamente en la resolución de diversos tipos de problemas de planificación tales como scheduling o routing (Chang et al, 2005; Gou et al, 2005; Jaskowski y Sobotka, 2006). Los AEs son metaheurísticas que comparten un concepto base común que es simular la evolución de los individuos que forman la población usando un conjunto de operadores predefinidos de selección y de búsqueda. Existe una gran variedad de AEs, dentro de ellos se incluyen los Algoritmos Genéticos (Goldberg, 1989) que son los utilizados en este problema. Tendencias actuales en AEs hacen uso de enfoques con multirecombinación (Eiben y Bäck, 1997; Eiben et al., 1994; Eiben et al., 1995) y enfoques con múltiples padres conocidos como MCMP (Multiple Crossover Multiple Parent) (Esquivel et al., 1997; Esquivel et al., 1998; Esquivel et al., 1999).

La herramienta presentada aquí, utiliza un algoritmo evolutivo con multirecombinación, que es el generador de múltiples soluciones. Se encuentra en la segunda fase de desarrollo del prototipo en la que se ha incorporado la replanificación de un conjunto de locaciones luego de ocurrida una contingencia o interrupción en la planificación original. El objetivo es mejorar las planificaciones y replanificaciones de mantenimiento de las locaciones petroleras.

\section{PROCEDIMIENTO}

\section{Descripción del problema}

Las empresas petroleras realizan visitas de mantenimiento preventivo a cada una de sus locaciones petroleras (pozos productores, inyectores, baterías y colectores). Un yacimiento está formado por bloques y a su vez éste por baterías. Cada batería está formada por pozos de producción que son en promedio entre 15 y 20 . Cada pozo tiene diferente nivel de producción que es conocido a priori y varía en el tiempo. La producción del pozo define la categoría y la cantidad de veces que debe visitarse al mes. Los pozos no pueden ser visitados más de una vez al día y dependiendo del tipo de pozo, existen ciertas tareas que se deben realizar. Cada tarea tiene asignado un determinado equipamiento necesario, una frecuencia de realización y un tiempo aproximado de su duración. 
Actualmente, el recorrido que realizan los encargados de las locaciones, se planifica en base a la experiencia de los mismos. La jornada laboral comienza a la mañana y se visitan las locaciones en dos turnos de tres horas. Luego de finalizado cada turno, el responsable debe regresar a la base de operaciones, realizar determinadas actividades administrativas y luego comenzar con el siguiente turno. El tiempo demandado en cada locación dependerá del tipo de la misma. Existen contingencias aleatorias que hacen que el plan de mantenimiento de un turno no se cumpla según lo planificado, produciendo la necesidad de replanificar las visitas. Cuando ocurre esto, cada responsable redefine el nuevo itinerario utilizando su experiencia.

PAE tiene por objetivos planificar las visitas a un conjunto de locaciones que: a) Minimice el tiempo total de visitas, es decir, encontrar la planificación que en menor tiempo recorra las locaciones incluyendo el tiempo de intervención en cada una de las mismas; b) Maximice la cantidad de visitas en un período de tiempo; por ejemplo, teniendo en cuenta un período de mantenimiento de un mes, que visite la mayor cantidad de veces las locaciones; c) Replanifique las visitas a partir de desviaciones en la planificación original. Frente a la ocurrencia de eventos externos que condicionan operativamente la ejecución de un plan de mantenimiento, proveer de planificaciones alternativas sin disminuir significativamente la calidad de las mismas.

\section{Formulación del problema de planificación}

El problema se puede definir como (Pinedo, 1995):

$1\left|s_{j k}\right| C_{\max }$

Denota un problema de scheduling de máquina única con $n$ tareas sujetas a tiempos de preparación dependientes de la secuencia. Donde las tareas a planificar son el servicio de mantenimiento (o intervención) en cada una de las locaciones petroleras. La función objetivo es minimizar el makespan $\left(C_{\max }\right)$ sujeto a los tiempos de preparación dependientes de la secuencia. El makespan puede calcularse como:

$\sum_{k=1}^{n} s_{j k}+t_{k}$

Donde existe un tiempo de traslado entre cada una de las locaciones al que se denomina $s_{j k}$, que representa el costo en tiempo de ir de la locación $j$ a la locación $k$ y $t_{k}$ es el tiempo de mantenimiento en la locación $k$.

\section{Un algoritmo multirecombinativo para $P A E$}

Para resolver el problema de planificación de recorrido de las locaciones petroleras, se utilizó un algoritmo evolutivo multirecombinativo. Para codificar adecuadamente las visitas a las locaciones petroleras que representan una posible solución, se utilizó una permutación $p=\left(p_{1}, p_{2}, \ldots, p_{n}\right)$, donde cada una de ellas, es un cromosoma en el cual $p_{i}$ representa la locación $i$ que debe ser visitada y $n$ representa la cantidad de locaciones a visitar. El cromosoma establece el orden de la secuencia a seguir para visitar cada locación. El algoritmo buscará la mejor permutación posible, a fin de obtener la planificación óptima que satisfaga los objetivos.

En la Figura 1 se puede observar la estructura del algoritmo evolutivo utilizado para este problema de planificación. El algoritmo crea una población inicial Stud(0) de sementales generados aleatoriamente que representan $\mu$ soluciones al problema y luego estas soluciones son evaluadas. Seguidamente, de la población de individuos denominados sementales, se selecciona un individuo a través de selección proporcional. Se genera un pool de apareamiento con n2 padres generados aleatoriamente (inmigrantes). El semental se aparea con cada padre inmigrante del pool de apareamiento y las parejas se someten a operaciones de recombinación, y se generan $2^{*} \mathrm{n} 2$ descendientes. El mejor de los $2^{*} \mathrm{n} 2$ descendientes, se almacena en un pool de hijos temporal. Esta operación de recombinación se repite n1 veces, para diferentes puntos de corte cada vez, hasta que 
el pool de hijos se complete. Finalmente, el mejor descendiente creado de n2 padres y n1 operaciones de recombinación, se inserta en la nueva población.

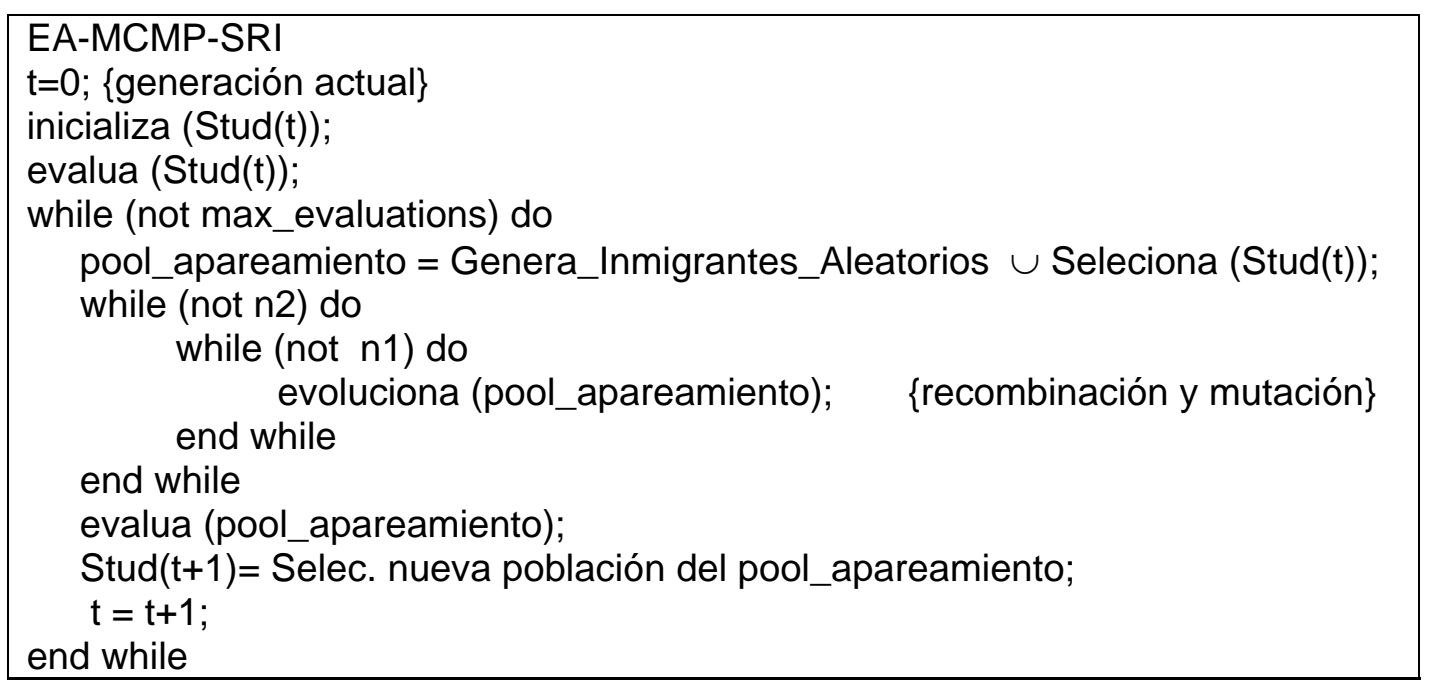

Fig. 1: Algoritmo Evolutivo para PAE

Este proceso es llamado MCMP-SRI y es una variante de multirecombinación (Pandolfi et al., 2002; Pandolfi et al., 2004). Fue aplicado en diferentes problemas de planificación de máquina única para casos estáticos y casos dinámicos y los resultados obtenidos fueron satisfactorios. El método de recombinación utilizado fue PMX (Partial Mapped Crossover). Este operador fue propuesto por (Goldberg y Lingle, 1987) y puede verse como una extensión del cruzamiento de dos puntos para representaciones basadas en permutaciones. La operación de mutación usada es llamada Mutación por intercambio (Swapping Mutation - SM), la cual selecciona dos posiciones aleatorias e intercambia los alelos. La selección de individuos fue a través del operador de selección proporcional.

\section{RESULTADOS Y DISCUSIÓN}

\section{Preparación de Datos}

Para resolver el problema fue necesario preparar los datos de entrada, ya que originalmente las distancias entre las locaciones petroleras no estaban procesadas. Se realizó el cálculo de las distancias entre las locaciones petroleras basados en el plano de caminos y distribución del yacimiento. Matemáticamente, es sabido que la distancia entre dos puntos que se encuentran en cualquier lugar del sistema de coordenadas, está determinada por la relación denominada distancia euclídea. No obstante, en este problema sólo se puede calcular la distancia entre dos puntos, teniendo en cuenta el camino que existe para llegar a ellos. Por esta razón se utilizó el plano de las ubicaciones de las locaciones y se escalaron las distancias entre las mismas.

\section{Diseño Experimental y Resultados}

Para realizar los experimentos se establecieron las siguientes suposiciones y restricciones al problema. Para la evaluación de la aplicación se trabajó con 110 locaciones petroleras correspondientes a un bloque de la zona norte de explotación. La velocidad de recorrido se estableció en 12 segundos cada 100 metros y se fijó el mismo tiempo de intervención utilizado en el mantenimiento efectivo para cada locación en el proceso de mantenimiento preventivo.

Para el algoritmo evolutivo propuesto se utilizó un tamaño de población de 15 individuos. La población inicial se generó aleatoriamente. El número máximo de evaluaciones es $2^{22}$. Se estableció la probabilidad de mutación en 0,05 y la probabilidad de recombinación en 0,65. El número n1 (número de operaciones de recombinación) y n2 (número de padres), se estableció en 16 y 18 respectivamente. Los parámetros (tamaño de la población, criterio de parada, probabilidades, etc.) se seleccionaron en base a la experimentación de los valores previamente usados con éxito (Lasso et al., 2004). La Tabla 1 resume los parámetros utilizados en todas las corridas. 
Para analizar la eficiencia del algoritmo propuesto, se realizaron dos tipos de experimentos. El primero con el objetivo de analizar la eficiencia del algoritmo para la planificación total del conjunto de locaciones, correspondientes al bloque norte de la zona de explotación (110 locaciones) y el segundo con el objetivo de analizar la eficiencia del algoritmo en cuanto a la replanificación de un subconjunto de esas 110 locaciones cuando se produce una interrupción en el proceso de mantenimiento, causando la imposibilidad de continuar con la planificación original.

En el primer experimento se realizaron 20 corridas independientes usando los valores de los parámetros resumidos en la Tabla 1.

Tabla 1: Parámetros del Algoritmo Evolutivo.

\begin{tabular}{|c|c|}
\hline Tamaño población & 15 \\
\hline Tamaño cromosoma (locaciones) & 110 \\
\hline Criterio parada (generación) & 500 \\
\hline Recombinación & PMX \\
\hline Mutación & SW \\
\hline Probabilidad Recombinación & 0,65 \\
\hline Probabilidad Mutación & 0,05 \\
\hline $\mathrm{N}^{\circ}$ de recombinación (n1) & 16 \\
\hline $\mathrm{N}^{\circ}$ de padres (n2) & 18 \\
\hline
\end{tabular}

En el segundo experimento se toma cada una de las planificaciones obtenidas en el primer experimento y para cada una se genera una interrupción en forma aleatoria. Se analizan los resultados de replanificar a partir de la interrupción en la planificación original de acuerdo a los siguientes tres tipos de acciones: 1) Las locaciones no visitadas en el turno interrumpido, se replanifican al final de la planificación original; 2) Se replanifican los turnos en el orden de la secuencia restante; 3) A partir de la locación interrumpida, se utiliza el algoritmo multirecombinativo para generar una nueva planificación con las locaciones restantes.

Los resultados obtenidos se compararon con información histórica facilitada por la empresa petrolera para una planificación tipo.

En la Tabla 2 se muestran los resultados obtenidos por el algoritmo en 20 corridas independientes, para las 110 locaciones petroleras del área de explotación. Se puede observar que en cada corrida la cantidad de turnos planificados es de 4 o 5 . El tiempo total planificado en cada una de las corridas para realizar las visitas a las 110 locaciones se encuentra entre 12 horas y 12:30 horas. El mínimo tiempo para una planificación se encontró en la corrida 18 donde se visitan las 110 en 717 minutos (11 horas, 57 minutos y 28 segundos) y se recorren 86,458 Km. El máximo tiempo planificado se encontró en la primera corrida donde se visitan las 110 locaciones en 753 minutos (12 horas, 33 minutos y 44 segundos) y se recorren $104,041 \mathrm{Km}$.

En la Tabla 3 se muestra una comparación de una planificación específica para el recorrido de 110 locaciones, realizada por la empresa petrolera y la planificación obtenida por PAE para la misma cantidad de locaciones. Se puede observar que PAE obtiene una planificación de los turnos que supera en un $50 \%$ o más a los turnos planificados por la empresa, es decir que mientras la planificación de la empresa visita 17 pozos PAE planifica la visita de 25. Por lo tanto, se utilizan menos turnos para visitar la misma cantidad de locaciones. También se puede ver que mientras la empresa planifica 3 días para visitar las 110 locaciones (6 turnos), PAE planifica las mismas locaciones en 2 días (4/5 turnos).

En general, mientras que la planificación original demanda un tiempo total de 18 horas 24 minutos, la mejor planificación provista por PAE demanda 11 horas 57 minutos, logrando un ahorro de 6 horas 27 minutos, algo más de 2 turnos sobre un total de 6 . 
El tiempo de procesamiento de una planificación típica (110 locaciones), en una PC Pentium 4 de 2.80 GHZ con $512 \mathrm{MB}$ de RAM, es en promedio, de 11 minutos.

Tabla 2: Resultados obtenidos por PAE.

\begin{tabular}{|c|c|c|c|c|}
\hline$N^{\circ}$ Corrida & Total Turnos & Km. Recorridos & Minutos & Tiempo Total \\
\hline 1 & 5 & 104,041 & 753 & $12: 33: 44$ \\
\hline 2 & 4 & 91,676 & 728 & $12: 08: 22$ \\
\hline 3 & 5 & 99,344 & 743 & $12: 23: 33$ \\
\hline 4 & 5 & 102,011 & 748 & $12: 28: 32$ \\
\hline 5 & 5 & 97,393 & 737 & $12: 17: 22$ \\
\hline 6 & 5 & 97,607 & 740 & $12: 20: 13$ \\
\hline 7 & 5 & 95,699 & 735 & $12: 15: 13$ \\
\hline 8 & 5 & 94,515 & 734 & $12: 14: 01$ \\
\hline 9 & 5 & 99,695 & 743 & $12: 23: 24$ \\
\hline 10 & 5 & 100,98 & 746 & $12: 26: 55$ \\
\hline 11 & 5 & 93,293 & 729 & 12:09:54 \\
\hline 12 & 5 & 98,636 & 742 & $12: 22: 04$ \\
\hline 13 & 5 & 99,162 & 743 & $12: 23: 02$ \\
\hline 14 & 5 & 103,313 & 750 & $12: 30: 21$ \\
\hline 15 & 4 & 92,4 & 729 & $12: 09: 47$ \\
\hline 16 & 5 & 97,564 & 740 & $12: 20: 15$ \\
\hline 17 & 5 & 101,354 & 747 & $12: 27: 22$ \\
\hline 18 & 4 & 86,458 & 717 & $11: 57: 28$ \\
\hline 19 & 5 & 97,803 & 740 & $12: 20: 32$ \\
\hline 20 & 4 & 89,289 & 721 & $12: 01: 49$ \\
\hline \multicolumn{2}{|c|}{ Mínimo } & 86,458 & 717 & \\
\hline \multicolumn{2}{|c|}{ Máximo } & 104,041 & 753 & \\
\hline
\end{tabular}

Tabla 3: Comparación Empresa Petrolera y PAE.

\begin{tabular}{|c|c|c|c|c|c|c|c|}
\hline \multicolumn{4}{|c|}{ Planificación Empresa Petrolera } & \multicolumn{4}{|c|}{ Planificación con PAE } \\
\hline Día & Turno & Pozos & Tiempo & Día & Turno & Pozos & Tiempo \\
\hline \multirow[t]{2}{*}{1} & 1 & 18 & 181,50 & 1 & 1 & 27 & 180,48 \\
\hline & 2 & 16 & 176,23 & & 2 & 27 & 181,41 \\
\hline \multirow[t]{2}{*}{2} & 3 & 18 & 184,20 & 2 & 3 & 29 & 184,52 \\
\hline & 4 & 20 & 182,20 & & 4 & 27 & 170,70 \\
\hline \multirow[t]{3}{*}{3} & 5 & 19 & 181,90 & & & 110 & 717,11 \\
\hline & 6 & 19 & 198,60 & & & & 11:57:28 \\
\hline & & 110 & 1104,63 & & & & \\
\hline & & & $18: 24: 38$ & & & & \\
\hline
\end{tabular}

En la tabla 4 se resumen los resultados obtenidos por PAE en el segundo experimento descrito anteriormente. Los aspectos más relevantes mostrados en esta comparación son los siguientes: el número de locaciones petroleras visitadas antes de que ocurra una interrupción (columna NBC), el número de locaciones petroleras a visitar luego de ocurrida la interrupción (columna NAC), tiempo total de la planificación original (columna Planif. Original), tiempo total de la planificación cuando al ocurrir una interrupción el algoritmo replanifica de acuerdo a la acción 1 (columna Acción 1), replanifica de acuerdo a la acción 2 (columna Acción 2), y replanifica de acuerdo a la acción 3 (columna Acción 3). Se puede observar que las replanificaciones varían entre un mínimo de 11 locaciones a 89 locaciones. La acción 1 es la menos favorables de las tres y la acción 2, que es la aplicada por el equipo de mantenimiento, si bien mejora a la anterior no lo hace para la acción de 
planificación con el algoritmo multirecombinativo. El máximo tiempo para una planificación realizando la acción 2, fue de 12 horas y 24 minutos, el mínimo tiempo fue de 11 horas y 54 minutos. Además en la mayoría de las corridas (19 de 20), la última acción mejora los resultados obtenidos en la planificación original. La razón de esta mejora está basada en el uso de un algoritmo específico para la replanificación (EA-MCMP-SRI) para un tamaño de problema menor a 110 locaciones.

Tabla 4: Resultados obtenidos por PAE en la replanificación.

\begin{tabular}{|c|c|c|c|c|c|c|}
\hline$N^{0}$ & NBC & NAC & Planif. & Acción1 & Acción2 & Acción3 \\
\hline 1 & 91 & 19 & $12: 33: 04$ & $12: 38: .35$ & $12: 27: 17$ & $12: 07: 22$ \\
\hline 2 & 21 & 89 & $12: 08: 22$ & $12: 11: 18$ & $12: 15: 54$ & $12: 04: 41$ \\
\hline 3 & 36 & 74 & $12: 23: 33$ & $12: 20: 56$ & $12: 20: 56$ & $12: 14: 51$ \\
\hline 4 & 33 & 77 & $12: 28: 32$ & $12: 31: 33$ & $12: 26: 03$ & $12: 09: 37$ \\
\hline 5 & 25 & 85 & $12: 17: 22$ & $12: 19: 25$ & $12: 14: 20$ & $11: 58: 37$ \\
\hline 6 & 70 & 40 & $12: 20: 13$ & $12: 19: 02$ & $12: 14: 40$ & $12: 04: 53$ \\
\hline 7 & 56 & 54 & $12: 15: 13$ & $12: 14: 39$ & $12: 13: 34$ & $12: 04: 57$ \\
\hline 8 & 34 & 76 & $12: 14: 01$ & $12: 16: 20$ & $12: 06: 54$ & $12: 01: 46$ \\
\hline 9 & 99 & 11 & $12: 23: 24$ & $12: 20: 06$ & $12: 20: 11$ & $12: 15: 17$ \\
\hline 10 & 44 & 66 & $12: 26: 55$ & $12: 25: 03$ & $12: 19: 28$ & $12: 12: 50$ \\
\hline 11 & 55 & 55 & $12: 09: 54$ & $12: 07: 58$ & $12: 15: 30$ & $11: 54: 00$ \\
\hline 12 & 58 & 52 & $12: 22: 04$ & $12: 18: 51$ & $12: 25: 48$ & $12: 02: 40$ \\
\hline 13 & 76 & 34 & $12: 23: 02$ & $12: 22: 15$ & $12: 14: 40$ & $12: 07: 49$ \\
\hline 14 & 51 & 59 & $12: 30: 21$ & $12: 27: 03$ & $12: 26: 54$ & $12: 21: 24$ \\
\hline 15 & 32 & 78 & $12: 09: 47$ & $12: 11: 13$ & $12: 16: 25$ & $12: 08: 40$ \\
\hline 16 & 55 & 55 & $12: 20: 15$ & $12: 19: 34$ & $12: 11: 55$ & $12: 11: 28$ \\
\hline 17 & 79 & 31 & $12: 27: 22$ & $12: 26: 06$ & $12: 28: 00$ & $12: 24: 51$ \\
\hline 18 & 51 & 59 & $11: 57: 28$ & $11: 53: 13$ & $12: 06: 48$ & $12: 00: 44$ \\
\hline 19 & 39 & 71 & $12: 20: 32$ & $12: 22: 15$ & $12: 23: 12$ & $12: 05: 16$ \\
\hline 20 & 35 & 75 & $12: 01: 49$ & $12: 03: 41$ & $12: 06: 50$ & $11: 58: 48$ \\
\hline
\end{tabular}

\section{CONCLUSIONES}

El problema de planificación tratado denota un problema de scheduling de máquina única, donde las tareas están sujetas a tiempos de preparación, dependientes de la secuencia. Los AEs son metaheurísticas poblacionales, probadamente eficientes en problemas de scheduling, ya que utilizan un buen balance entre una exploración del espacio de búsqueda y la explotación de los sub-esquemas que se encuentran codificados dentro de las soluciones. PAE es una aplicación que utiliza un algoritmo evolutivo multirecombinativo, llamado MCMP-SRI, que resulta una herramienta eficaz para la planificación dinámica del mantenimiento de locaciones petroleras.

Del análisis y las comparaciones realizadas con los planes de mantenimiento ejecutados, PAE ofrece las siguientes ventajas comparativas: 1) Presenta planificaciones que mejoran el plan de mantenimiento producido por expertos, reduciendo hasta una tercera parte del tiempo total, con la correspondiente reducción de costos; 2) Provee múltiples soluciones en diferentes corridas independientes. A menudo una solución mejor (plan de mantenimiento) no puede ejecutarse por determinadas condiciones operativas, por lo tanto es necesario seleccionar otra que si bien puede no ser tan buena como la anterior es factible de ejecutarse; 3) Frente a la ocurrencia de contingencias en la planificación original, PAE replanifica las locaciones, tratándolas como si fueran una nueva planificación en lugar de continuar con la planificación original, lo cual sería usualmente ineficiente en estas condiciones.

\section{AGRADECIMIENTOS}

A la Universidad Nacional de la Patagonia Austral por su apoyo al grupo de investigación y la cooperación. 


\section{REFERENCIAS}

Brucker P.; "Scheduling Algorithms”, Springer-Verlag, $3^{\text {ra }}$ edición, New York (2004).

Chang P., J. Hsieh e Y. Wang; "Genetic Algorithm and Case-Based Reasoning Applied in Production Scheduling", Knowledge Incorporation in Evolutionary Computation, Springer, 215-236 (2005).

Eiben A.E. y Th. Bäck; "An Empirical Investigation of Multi-Parent Recombination Operators in Evolution Strategies", Evolutionary Computation: 5(3), 347-365 (1997).

Eiben A.E., P.E. Raué y Z. Ruttkay; "Genetic algorithms with multi-parent recombination", Proc. 3rd Conference on Parallel Problem Solving from Nature, Springer-Verlag, number 866 in LNCS, 7887 (1994).

Eiben A.E., C.H.M. Van Kemenade, y J.N. Kok; "Orgy in the Computer: Multi-Parent Reproduction in Genetic Algorithms", Proc. 3rd European Conference on Artificial Life, Springer-Verlag, number 929 in LNAI, pages 934-945 (1995).

Esquivel S., A. Leiva y R. Gallard; "Multiple Crossover per Couple in Genetic Algorithms", Proc. Fourth IEEE Conference on Evolutionary Computation (ICEC'97), pp 103-106, Indianapolis, USA April (1997).

Esquivel S., A. Leiva y R. Gallard; "Couple Fitness Based Selection with Multiple Crossover per Couple in Genetic Algorithms", Proc. International Symposium on Engineering of Intelligent Systems (EIS'98), pp 235-241, La Laguna, Tenerife, Spain, February (1998).

Esquivel S., H. Leiva y R. Gallard; "Multiple Crossovers between Multiple Parents to Improve Search in Evolutionary Algorithms", Proc.Congress on Evolutionary Computation (IEEE), pp 1589-1594, Washington DC (1999).

Goldberg. D; "Genetic Algorithms in Search, Optimization, and Machine Learning", pp 60-85, Addison Wesley Logman, USA (1989).

Goldberg, D. y R. Lingle; "Alleles, loci and the traveling salesman problem", Proc. of the First International Conference on Genetic Algorithms, Lawrence Eribaum Associates, pp. 154-159, Hillsdale, NJ (1987).

Guo, Y., L. Suhl y M.P. Thiel; "Solving the Airline Crew Recovery Problem by a Genetic Algorithm with Local Improvement", Operational Research an International Journal: 5(2) (2005).

Jaskowski P. y A. Sobotka; "Multicriteria Construction Project Scheduling Method using Evolutionary Algorithm", Operational Research an International Journal: 6(3) (2006).

Lasso M. y otros cuatro autores; "Solving Dynamic Tardiness Problems in Single Machine Environments"; Congress on Evolutionary Computation CEC '04, Portland U.S.A, 1, 1143-1149 (2004).

Pandolfi D. y otros cuatro autores; "Studs Mating Immigrants in Evolutionary Algorithm to Solve the Earliness-Tardiness Scheduling Problem", Cybernetics and Systems of Taylor and Francis Journal: 33(4), 391-400 (2002).

Pandolfi D. y otros cuatro autores; "Knowledge Insertion: an Efficient Approach to Reduce Search Effort in Evolutionary Scheduling", Journal of Computer Science \& Technology: 4(2), 109-114 (2004).

Pinedo M.; "Scheduling: Theory, Algorithms and System", First edition, pp 48-56, Prentice Hall, Englewood Cliffs, New Jersey (1995). 